\title{
CEMENT LEAKAGE FOLLOWING PERCUTANEOUS VERTEBRAL AUGMENTATION: CASE REPORT
}

Tran Huy Hung*, Nguyen Minh Tien*

\section{ABSTRACT}

Background: Percutaneous vertebroplasty (PV) has become an important minimally invasive surgical technique for vertebral compression fractures. However, indications for $\mathrm{PV}$ in these cases due to trauma had many rivals and unclearly. These complications, especially cement leakage still common, has many severe consequent. Object and method research: A 60-years-old man, who underwent vertebroplasty in low medical level. He had complete paralised of two leg, urine retention, and severe low back pain. We examined clinical signs, imaging, find and compared with literatures. Case presentation and discussion: We present a case, 60-years-old man, had labor accident, after that he could returned to state of normality. At the second day, he went to local hospital, had diagnosis: compression fractures of L1-L2. He underwent percutaneous vertebroplasty of L1-2 under local anesthesia. Post-operative, he had complete paralised of two leg, urine retention, and severe low back pain. He had exmined again, MRI and took second surgery to removed part of cement leakage. After the second surgery, radiological showed they had complete removed cement leakage, but the patient's recovery was uneventful and stable. The patient was hospitalized at our instituation after 03 day with complaints of severe low back pain, urine retention and complete paralised of two leg. Conclusion: This is basic technique, widely and rewarding; also has many severe incident, complications. Consequently, we suggest that

\footnotetext{
* 108 Military Central Hospital

Responsible person: Tran Huy Hung

Email: hungth.ss108@gmail.com

Date of receipt: $31 / 5 / 2021$

Date of scientific judgment: 05/7/2021

Reviewed date: 10/8/2021
}

this technique have to perform in right indications, in the modern medical centre and fully trained surgeon.

Key words: Vertebroplasty, compression fractures, cement leakage.

\section{BACKGROUND}

Vertebral compression fractures is cause of persistent pain in lower back, spinal defomity and injuries root of spinal cord. Percutaenous augmentation technique has confirmed and widely used on the world in treament vertebral compression fractures (VCFs) [1]. However, indication of this technique on VCF due to trauma has many argue and unclearly. Moreover, this technique has many complications, and the most common of which is cement leakcage. Although most cases are asymptomatic, some severe cases of cement leakage into the spinal canal, dura, or intervertebral foramen may cause pulmonary or vein embolism, and can be life-threatening [2]. Base on our experienced, cement leakcage into spinal canal is not rarely, but it caused both leg completed paralized is rarely. Therefore, we present one case 60-year-old man, has underwent vertebralplasty in low level hospital. Post-operative, he had cement leakcage into spinal canal. $\mathrm{He}$ had to underwent secondary surgery to taked out part of cement leakcage but there is not any progress. The patient was hospitalized at our institution again due to severe of low back pain, both leg completed paralized, urinary incontinence at $3^{\text {rd }}$ day after VP procedures. 


\section{CASE PRESENTATION}

One case, 60-year-old man, he had traffic accident and first-aid at low level hospital; was diagnosed compression fractures L1-2; underwent vertebralplasty. After operated, he

had more low back pain, both leg complete paralysed. He was re-examined, x-ray and secondary surgery to took out part of cement leakcage but had not any progress. (Fig 1).
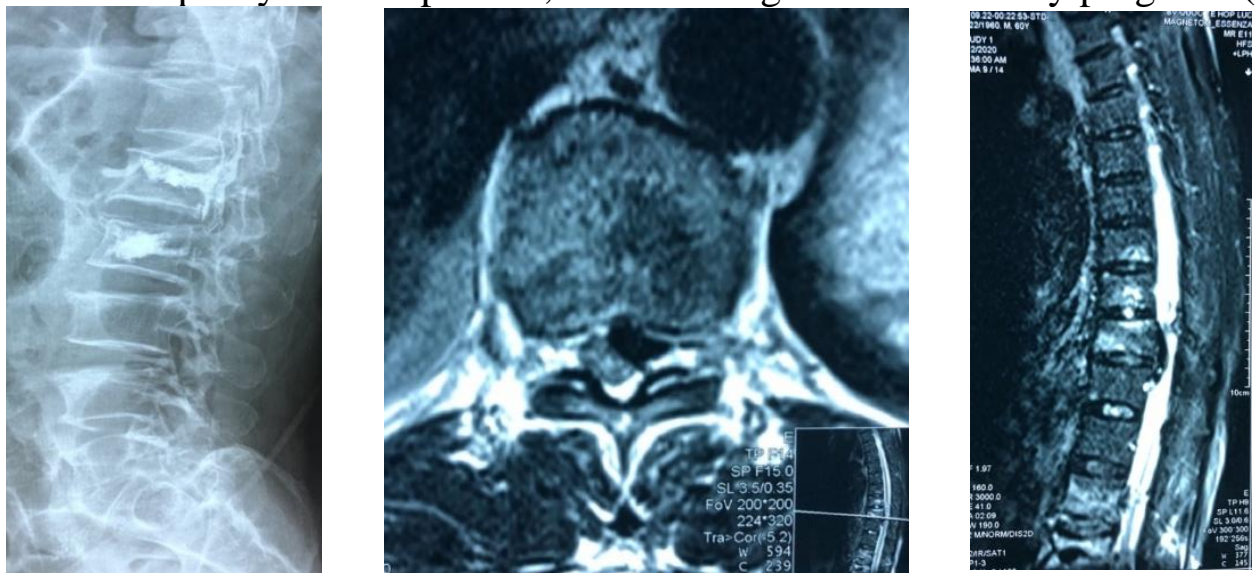

Fig1. XR and MRI post 1st-operative.

The patient was hospitalized at our hospital again due to progression of low back pain, completed paralysed. The pain was severe and stabbing, and was aggravated by position changes; decrease sensation of lateral of both thigh, pubis. Base on MRI, X-

ray after operatived (Fig1) with clinical symtom; we diagnosed he had stenosis of L12 due to cement leakcage after VP. However, MRI after secondary operatived show that they took out all cement leakcage and decompression spinal canal. (Fig 2).
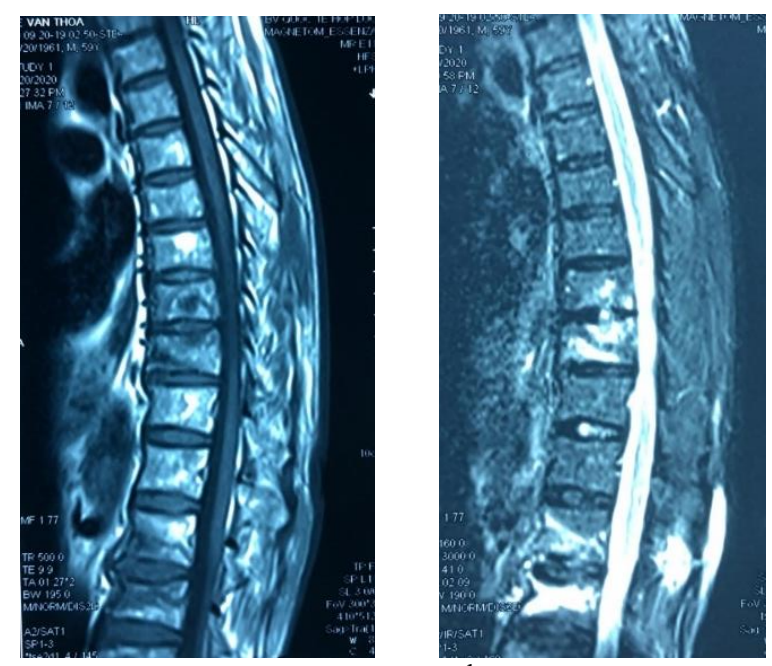

Fig2. MRI Post- $2^{\text {nd }}$ operative

We checked content of $2^{\text {nd }}$ surgery and saw that our colleagues at low level hospital removed lamina $\mathrm{T} 10$, pedicle and lamina $\mathrm{T} 11$, part of $\mathrm{T} 12$; cut both root of $\mathrm{T} 11$. any harm to epidural. What a pity that we do not have content of $1^{\text {st }}$ surgery (VP) to find causes of this complication. What problem and when it happening. 


\section{DISSCUSSION}

Percutaneous vertebroplasty (PVP) is a minimally invasive procedure widely used for the treatment of pain due to vertebral compression fractures of the spine. Reports on the outcome for vertebroplasty have suggested that most patients experience partial or complete pain by restoring the vertebra height and injecting cement into the fractured bone $(3,4)$. However, it is still surgery and has some risks (5). The most frequent complication is cement leakage (6), the complication rate for vertebroplasty is estimated from $5 \%$ to $87 \%$ (7).

Cement leakage complication can occur when bone cement leaks into the spinal canal, affecting foraminal area or epidural space which could increase pain, tingling, numbness, and or weakness, as well as possibly cause problems with function and movement. There have also been reported cases of bone cement getting into a vertebral vein and causing pulmonary embolism. Hence, it is important to closely monitored to avoid leakage during cement injection. Risk factors for cement leakage in percutaneous vertebroplasty have been studied previously, which are the characteristics of vertebral compression fractures and distribution of bone cement in vertebral body, volume of injected bone cement, injection technique, adhesion of bone cement (2).

In this case study, the patient presented to our hosptial with a medical history of percutaneous vertebroplasty treatment. The diagnosis of this patient is vertebral compression fracture due to traffic accident in local hospital. He reported after having traffic accident, he was still able to go home by himself. He went to local hospital to check his status on the second day of accident. There was no radiography about his vertebral compression fracture before performing vertebroplasty. It was only a post-vertebroplasty X-ray when he arrived to our hospital. Through medical history and post-vertebroplasty X-ray, we think that this patient was a middle age, male patient with mild and stable vertebral compression fracture. With all these factors, conservative treatment is acceptable in this patient. Unfortunately, this patient was indicated to perform vertebroplasty without any conservative treatment methods such as brace, bed rest, analgesic drug,...

There is still debate over the indication of vertebroplasty in vertebral compression fractures treatment. Khan's study showed that the indication of vertebroplasty should fulfill 3 criteria such as compression vertebral body fracture or deformity, mechanical back pain from the fracture level, and failed initial conservative treatment. The failure of medical therapy is defined as pain persisting at a vertebral body fracture level that prevents ambulation despite 24 to 48 hours of potent analgesic therapy; with no response to bed rest, bracing, analgesic therapy with no response to physical therapy and having unacceptable side effects due to the analgesic therapy necessary to reduce pain to a tolerable level (8). To our best knowledge, performing vertebroplasty for those patients is unnecessary and may cause the great economic loss for patients, especially for those patients are common labor who have no finacial funding.

Because of lacking the information of previous vertebroplasty, therefore the reason of cement leakage are still unclear. We hypothesized the reason of cement leakage was a result of either inexperience surgeons during performing this technique. Besides, in 
patient presented with mild compression fracture and high density of bone, no sign of osteoporosis, the short bone cement injection timing and lacking bone cement adhesive strength are the main reasons causing bone cement leakage into spinal canal. The average volume of the injected cement was 4-5ml for thoracolumbar fracture lesion (8). Over the average volume of the injected cement within short injection timing also is the major reason of cement leakage in this case.

Identify whether bone cement leakage during vertebroplasty is a mandatory process. Moreover, final postprocedure images should be obtained to confirm optimal cement positioning within the vertebral body before ending surgery (8). Injection should also be stopped if cement reaches the posterior part of the vertebra. In this case, bone cement leakage was present in the central canal which was mechanical compression of the spinal cord. The initial symptoms of paraplegia and urinary incontinence might be occurred resulting of long-time compression. After vertebroplasty, patient's clinical status and final postprocedure images of L1, L2 vertebral body had not been checked. Till to the second day post-op, the patient was undergone surgical decompression and removal of epidural cement. $\mathrm{Li}$ et al. indicated that vertebroplasty should be performed after fulfilling 3 criteria of indication. The intraoperative computed tomography-guided navigation and neuromuscular monitor are helpful during vertebroplasty (2). When bone cement leakage happened causing damage of nerve root, vertebroplasty should be withdrawn and patients supposed to be undergone surgical decompression immediately in order to avoid second lesion.
According to the previous vertebroplasty record, bone cement leaked into spinal canal, moved to the above of cement injection level affecting foraminal area. Therefore, the posterior lamina of T10-T12 and pedicle of T11, T12 were resected extensively. T11 nerve root was also removed due to the great compression of cement leakage. It also caused corresponding clinical manifestations

After additional decompression surgery, bone cement leakage part was completely removal without spinal dura matter's tear, but the clinical outcome was not improved. It suggested that the damage due to cement leakage may cause unrecoverable damage in a short time. Therefore, it supposed to be taken immediately action when cement leakage to avoid serious complications.

\section{CONCLUSION}

We reported a rare case of bone cement leakage causing symptoms of paraplegia and urinary incontinence in vertebroplasty patient. There is no indication of vertebroplasty in this case. The patient should be treated as conservative methods. We recommend that percutaneous vertebroplasty is an efficient technique with low complication rates and a significant reduction in pain. Nevertheless, cement leakage is not rare complication, thus this technique should be perform by experienced surgeon.

\section{REFERENCES}

1. Filippiadis DK, et al. Percutaneous Vertebroplasty and Kyphoplasty: current status, new developments and old controversies. Cardiovasc Intervent Radiol. 2017;40(12):1815-23.

2. Ziquan Li et al. Cement leakage following percutaneous kyphoplasty in a 
patient after a posterior lumbar fusion: a case report; MC Surgery (2020) 20:74.

3. Qi Y, et al. Comparison of percutaneous Kyphoplasty versus modified percutaneous Kyphoplasty for treatment of osteoporotic vertebral compression fractures. World Neurosurg. 2019;122:e1020-7.

4. Lamy O, Uebelhart B, Aubry-Rozier B. Risks and benefits of percutaneous vertebroplasty or kyphoplasty in the management of osteoporotic vertebral fractures. Osteoporos Int. 2014;25:807-19.

5. Kita K, et al. Surgical Removal of Circumferentially Leaked Polymethyl Methacrylate in the Epidural Space of the
Thoracic Spine after Percutaneous Vertebroplasty. Surg J (N Y). 2017;10:e1-5.

6. Lee JH, Lee JH, Jin Y. Surgical techniques and clinical evidence of vertebroplasty and kyphoplasty for osteoporotic vertebral fractures. Osteoporos Sarcopenia. 2017;3:82-9.

7. Ziquan $\mathbf{L i}$ et al, Cement leakage following percutaneous kyphoplasty in a patient after a posterior lumbar fusion: a case report. BMC Surgery (2020) 20:74

8. Majid Khan et al,. Percutaneous Vertebral Body Augmentations The State of Art, Neuroimag Clin N Am 29 (2019) 495-513 